\title{
SISTEMA DE MEDIÇÃO DE DESEMPENHO: UMA REVISÃO TERCIÁRIA DA LITERATURA
}

\author{
Gabriel Simões de Oliveira \\ Departamento de Engenharia Industrial - PUC Rio \\ Rua Marquês de São Vicente, 225 - Gávea, Rio de Janeiro - RJ \\ gsimoeso.25@gmail.com \\ Luiz Felipe Roris Rodriguez Scavarda do Carmo \\ Departamento de Engenharia Industrial - PUC Rio \\ Rua Marquês de São Vicente, 225 - Gávea, Rio de Janeiro - RJ \\ lf.scavarda@puc-rio.br \\ Edson da Costa Pinto Junior \\ Departamento de Engenharia Industrial - PUC Rio \\ Rua Marquês de São Vicente, 225 - Gávea, Rio de Janeiro - RJ \\ edsoncostap@hotmail.com
}

\begin{abstract}
RESUMO
Com o aumento da competição entre as organizações e elevação da complexidade das operações, a medição de desempenho torna-se uma importante ferramenta para auxiliar as tomadas de decisões. Então, a aplicação de um sistema de medição de desempenho (SMD) ganhou destaque entre os acadêmicos e no ambiente corporativo. Consequentemente, diversos estudos sobre Revisões Sistemáticas da Literatura foram realizados. Entretanto, há uma carência de estudos que integrem as visões das Revisões Sistemáticas da Literatura sobre a perspectiva do SMD. Neste contexto, este artigo tem como objetivo realizar uma primeira Revisão Terciária da Literatura, a fim de identificar como este tema tem sido abordado pela academia através de análise dos objetivos e gaps das pesquisas. A Revisão Terciária da Literatura foi executada a partir da análise de 22 artigos onde foi possível detectar uma necessidade em estudar modelos que auxiliem na definição de indicadores de desempenho e na compreensão do SMD, além de observar uma lacuna na abordagem das fases do ciclo de vida do SMD posteriores ao seu desenho.
\end{abstract}

Palavra-chave: Indicador de desempenho; Gerência de operações; Processos de negócios.

\begin{abstract}
With increasing competition between organizations and increasing complexity of operations, performance measurement becomes an important tool to assist decision making. Then, the usage of a performance measurement system (SMD) gained notoriety among academics and the corporate environment. Consequently, several studies on Systematic Literature Reviews have been conducted. However, there is a lack of studies that integrate the views of Systematic Literature Reviews from the perspective of SMD. In this context, this article aims to conduct a first Tertiary Literature Review, in order to identify how this
\end{abstract}


theme has been approached by academia through analysis of research objectives and gaps. The Tertiary Literature Review was performed from the analysis of 22 articles where it was possible to detect a need to study models that help in defining performance indicators and understanding the SMD, besides to find a gap in the approach of life cycle phases of SMD after your design.

Keywords: Performance indicator; Operations management; Business processes.

\section{Como Citar:}

OLIVEIRA, Gabriel Simões; SCAVARDA, Luiz Felipe; PINTO JUNIOR, Edson da Costa. Sistema de Medição de Desempenho: Uma Revisão Terciária da Literatura. In: SIMPÓSIO DE PESQUISA OPERACIONAL E LOGÍSTICA DA MARINHA, 19., 2019, Rio de Janeiro, RJ. Anais [...]. Rio de Janeiro: Centro de Análises de Sistemas Navais, 2019.

\section{INTRODUÇÃO}

O Sistema de Medição de Desempenho (SMD) é um sistema que engloba um conjunto de medidas de desempenho com a finalidade de quantificar a eficiência e eficácia das atividades da organização (NEELY, GREGORY e PLATTS, 1995). Para Neely et al. (1996), a eficácia representa a maneira na qual os requisitos dos clientes são atendidos e eficiência pode ser entendida em quão economicamente os recursos são utilizados para alcançar um determinado nível de satisfação do cliente. Lohman et al. (2004) entendem a medição de desempenho como uma atividade executada pelos gestores, a fim de atingirem metas predefinidas derivadas dos objetivos estratégicos da empresa. As realizações das medições de desempenho são executadas através de métricas, nas quais constituem as medidas de desempenho. Gunasekaran et al. (2001) afirmam que as métricas são necessárias para testar e verificar a viabilidade da estratégia traçada.

As medidas de desempenho devem ser capazes de medir o que representa a essência da organização, proporcionando uma relação no SMD entre medidas financeiras e nãofinanceiras para tomadas de decisão nos níveis estratégico, tático e operacional da organização (GUNASEKARAN, PATEL e MCGAUGHEY, 2004). Kennerly e Neely (2002) compreendem que a medida de desempenho deve sempre ser revista e atualizada para manter o nível de competitividade da organização. As medidas de desempenho podem ser: quantitativa ou qualitativa; financeira ou não-financeira; e avaliar a qualidade, flexibilidade, tempo e inovação (BRAZ, SCAVARDA e MARTINS, 2011). Para estes autores, uma boa medição de desempenho deve ser quantitativa e objetiva, ou seja, medir apenas o que é necessário; ser de fácil e rápida compreensão, tanto para o entendimento, como para a obtenção do resultado; multi-dimensional, evidenciando os trade-offs; ter inputs e outputs; e ter uma boa relação entre custo e benefício.

Com o aumento da concorrência nas organizações e, consequentemente, busca por melhorias, a medição de desempenho ganhou notoriedade, tanto na academia como na indústria (KEATHLEY-HERRING, 2017), logo diversas pesquisas foram publicadas neste contexto e muitos frameworks foram desenvolvidos ao longo dos anos (GUTIERREZ et al., 2015). Com o aumento da maturidade do tema, o SMD passou a ser utilizado em outras áreas, como, por exemplo, na Gestão da Cadeia de Suprimentos (MAESTRINI et al., 2017). Estes autores afirmam que, atualmente, as concorrências nas organizações expandiram para fora de suas fronteiras, elevando a competição para a coordenação do uso de recursos ao longo de toda cadeia, portanto, é imprescindível a mensuração do desempenho para garantir uma melhor governança e, consequentemente, melhores tomadas de decisões. Goshu e Kitaw 
(2017) destacam o aumento do interesse sobre o entendimento em como as organizações são medidas e como conseguem extrair valor destas medições através dos dados coletados. $\mathrm{O}$ crescimento do interesse em SMD é refletido no aumento do número de revisões da literatura sobre o tema. Neste sentido, o tema encontra-se maduro para uma primeira revisão terciária da literatura. Segundo Torgerson et al. (2018), a revisão terciária da literatura é útil quando existem várias revisões sistemáticas sobre um determinado tema, a fim de explorar a consistência entre os resultados das análises individuais das revisões, fornecendo uma síntese mais completa sobre a abordagem do tema.

Sendo assim, este artigo se propõe a realizar uma revisão terciária da literatura em Sistemas de Medição de Desempenho, a fim de identificar como este tema tem sido abordado pela academia através de análise dos objetivos e gaps das pesquisas. Sendo assim, duas perguntas de pesquisa norteiam este artigo: P1 Quais são os objetivos das revisões da literatura de SMD? P2 Quais são os ga1ps remanescentes das revisões de literatura em SMD?

Para alcançar o objetivo deste artigo, a metodologia aplicada foi baseada na abordagem apresentada por Thomé et al. (2016), onde são explicitadas as etapas para conduzir uma revisão da literatura em Gerência de Operações.

Este artigo foi estruturado em quatro seções: A primeira é a introdução, na qual foram apresentadas as motivações, maturidade das pesquisas em revisões da literatura para SMD e os objetivos. A segunda seção refere-se a metodologia utilizada na revisão terciária da literatura. A terceira seção desenvolve a análise dos resultados obtidos com a revisão terciária. A quarta seção traz as conclusões.

\section{METODOLOGIA}

Segundo Thomé et al. (2016), a revisão da literatura pode ser utilizada em situações onde são desejadas responder perguntas específicas da pesquisa; identificar os estudos existentes acerca de um determinado tema; selecionar e avaliar as contribuições da pesquisa; analisar e sintetizar os dados; evidenciar o caminho utilizado para se alcançar as conclusões. Logo, entende-se que a revisão da literatura é uma ferramenta específica para realizar uma análise crítica da literatura disponível.

Para conduzir esta revisão terciária da literatura a metodologia empregada foi dividida em quatro passos, originalmente adaptada da abordagem metodológica na condução de uma revisão da literatura em Thomé et al. (2016) realizada em oito passos. A relação entre os passos desta metodologia com a metodologia aplicada em Thomé et al. (2016), pode ser observada na Tabela 1.

O primeiro passo consiste na fase de planejamento, onde foi definido o protocolo de pesquisa com a estrutura da pesquisa, motivação, problematização e perguntas de pesquisa. No segundo passo da metodologia foi definida a base de dados Web of Science (WoS) e, então, foi realizada uma busca sobre o tópico "performance measurement system" sem nenhum tipo de restrição - obtendo um retorno de 836 trabalhos. A partir desta amostra, foram utilizadas keywords, a fim de refinar a pesquisa e, assim, obter papers específicos de revisão da literatura em SMD. As keywords aplicadas foram baseadas no protocolo de pesquisa do trabalho de Thomé et al. (2016) que apontam os termos mais comuns associados a revisão da literatura, em inglês: research synthesis; systematic review; evidence synthesis; research review; literature review; meta-analysis; meta-synthesis; mixed-method synthesis; narrative reviews; realist synthesis; meta-ethnography; state-of-the-art; rapid review; critical review; expert review; e conceptual review.

Após a aplicação do refino na pesquisa, foram obtidos 50 papers do tipo article (25), proceeding paper (17) e review (8). Conforme González-Albo e Bordons (2011), 
normalmente os trabalhos do tipo proceeding paper têm menor nível de maturidade em relação aos trabalhos do tipo article, trabalhos que passam por um processo de seleção mais rigoroso. Desta forma, todos os trabalhos de proceeding paper foram excluídos da amostra, enxugando a quantidade de trabalhos para 33 papers. A avaliação dos papers remanescentes foi feita através da aplicação de critérios de inclusão/exclusão, que consistem em aceitar apenas trabalhos em inglês, tópico principal da pesquisa em SMD e metodologia de pesquisa unicamente em revisão sistemática da literatura.

Os tópicos dos 33 artigos (autor, título, ano e resumo) foram extraídos para uma tabela em Excel, facilitando a aplicação dos critérios durante a leitura preliminar dos abstracts. Após a primeira etapa de inclusão/exclusão de papers, foi realizada a leitura fulltext com a finalidade de confirmar se um determinado artigo deveria ser incluído ou não na amostra. Ao final destas análises foram selecionados 13 artigos, então, a partir desta gama de trabalhos extraídos da base WoS, foi aplicada a técnica de snowballing, com intuito de enriquecer o estudo através de novas buscas para seleção de artigos de revisão da literatura sobre SMD. Esta técnica se propõe a usar a lista de referência de um artigo ou citações para identificar novos documentos e complementá-las de maneira sistemática (WOHLIN, 2014). Esta técnica foi realizada em duas etapas, conforme indicado por Wohlin (2014). A primeira etapa consiste em realizar buscas através das referências dos 13 artigos selecionados e é denominada como backward snowballing. A segunda etapa consiste em realizar buscas na base WoS para levantar trabalhos de revisão da literatura em SMD que citaram pelo menos um dos 13 artigos, sendo denominada de forward snowballing.

Para as etapas de backward e forward snowballing, os mesmos critérios de inclusão/ exclusão usados na análise anterior foram aplicados. No backward snowballing o resultado foi a adição de 8 artigos, já para o forward snowballing, o resultado foi o acréscimo de 1 artigo. Sendo assim, o total da amostra utilizada no presente estudo foi de 22 papers.

O terceiro passo da metodologia consiste na análise dos 22 artigos, sobretudo no campo dos objetivos e gaps de cada trabalho. Esta etapa contou com a análise de conteúdo baseado no trabalho de Seuring e Gold (2012). Por fim o quarto e último passo da metodologia consiste em expor de maneira integrada e organizada a síntese das análises extraídas. Para uma melhor compreensão da metodologia empregada nesta pesquisa, a Figura 1 apresenta os passos de forma sequencial com seus respectivos resultados.

TABELA 1 - Passos da Revisão terciária.

\begin{tabular}{|c|c|c|}
\hline Passos & Metodologia utilizada & Metodologia Thomé et al. (2016) \\
\hline 1 & $\begin{array}{l}\text { - Protocolo de pesquisa } \\
\text { - Formulação do problema } \\
\text { - Perguntas de Pesquisa }\end{array}$ & Passo 1 \\
\hline 2 & $\begin{array}{l}\text { - Seleção da base de dados } \\
\text { - Seleção keywords } \\
\text { - Uso de critérios de inclusão/exclusão } \\
\text { - Snowball } \\
\text { - Seleção de paper } \\
\text { - Análise qualitativa da coleta } \\
\text { - Verificar confiabilidade da pesquisa }\end{array}$ & $\begin{array}{l}\text { Passo } 2 \\
\text { Passo } 3 \\
\text { Passo } 4\end{array}$ \\
\hline 3 & - Análise e interpretação & $\begin{array}{l}\text { Passo } 5 \\
\text { Passo } 6\end{array}$ \\
\hline 4 & - Apresentação dos resultados & $\begin{array}{l}\text { Passo } 7 \\
\text { Passo } 8\end{array}$ \\
\hline
\end{tabular}

Fonte: Autor 


\begin{tabular}{|c|c|c|c|}
\hline Passo 1 & Passo 2 & Passo 3 & Passo 4 \\
\hline Planejamento & Coleta & Análise e interpretação & Resultado \\
\hline Retorno & Retorno & Retorno & Retorno \\
\hline - Escopo & - Base de dados & - Análise dos objetivos & - Integração das \\
\hline - Perguntas de pesquisa & - Palavras-chave & - Análise dos gaps & revisões sistemáticas \\
\hline & $\begin{array}{l}\text { - Critérios de } \\
\text { inclusão/exclusão } \\
\text { - Snowballing }\end{array}$ & & \\
\hline $\begin{array}{l}\text { Identificação do } \\
\text { escopo de } \\
\text { pesquisa }\end{array}$ & $\begin{array}{c}836 \text { papers } \\
\text { encontrados } \\
\text { (22 selecionados) }\end{array}$ & $\begin{array}{c}\text { Tabelas com } \\
\text { objetivos e gaps }\end{array}$ & $\begin{array}{l}\text { Síntese dos } \\
\text { resultados }\end{array}$ \\
\hline
\end{tabular}

FIGURA 1 - Resumo da metodologia. Fonte: Autor

\section{ANÁLISES E RESULTADOS}

Para obtenção de uma visão integrada sobre as perspectivas das revisões da literatura acerca do SMD, a Tabela 2 foi elaborada contemplando os 22 papers selecionados para este estudo, sendo organizada da seguinte forma: autor, título, periódico, palavra-chave, base de dados, tamanho da amostra e período de levantamento da amostra. Os campos preenchidos com N/D significam que os dados em referência não foram disponibilizados.

Através desta tabela, algumas informações relevantes para a compreensão das revisões da literatura em SMD foram observadas. O intervalo de publicação destes trabalhos foi compreendido entre 2005 e 2018, sendo o trabalho de Taticchi et al. (2010) o artigo com o maior período de amostragem em sua revisão (1970 a 2008) e, também, com o maior número de análises (6618 papers).

Além disto foi possível observar que as principais bases de dados adotadas para as pesquisas foram: Science Direct (9); EBSCO (8); e ABI/Inform Proquest (6). No que tange aos periódicos mais utilizados, constatou-se que o Business Process Management Journal (3) e o Measuring Business Excellence (3) foram os mais recorrentes, porém, não sendo suficiente afirmar que há uma preferência entre os periódicos para publicações desta natureza.

Para análise dos objetivos de cada artigo, a Tabela 3 foi estruturada de forma que possibilite a identificação dos principais tópicos estudados pelas revisões, sendo o modelo do Balanced Scorecard desenvolvido por Kaplan e Norton (1992) o de maior destaque entre as revisões. Contudo, outros importantes objetivos, também, podem ser observados, tais como o desenvolvimento de um modelo/framework para assistir na compreensão do SMD, auxiliar no estabelecimento das medidas de desempenho na fase de design ou dar suporte na implementação do sistema - seja em pequenas ou médias empresas ou no gerenciamento de processos de negócio.

Na Tabela 4, são apresentados os gaps apontados em cada revisão. Após uma análise destas lacunas, foi possível identificar que os principais campos em aberto estão relacionados a integração do SMD com outros sistemas e requisitos que impactam no sucesso de sua utilização. Além disso, há uma carência de trabalhos que validem a aplicação do SMD em um ambiente real e em outras culturas através de estudos empíricos. Outro gap que pode ser destacado nas pesquisas é a falta de consenso entre os acadêmicos em relação a qualidade da dimensão das medidas de desempenho. 


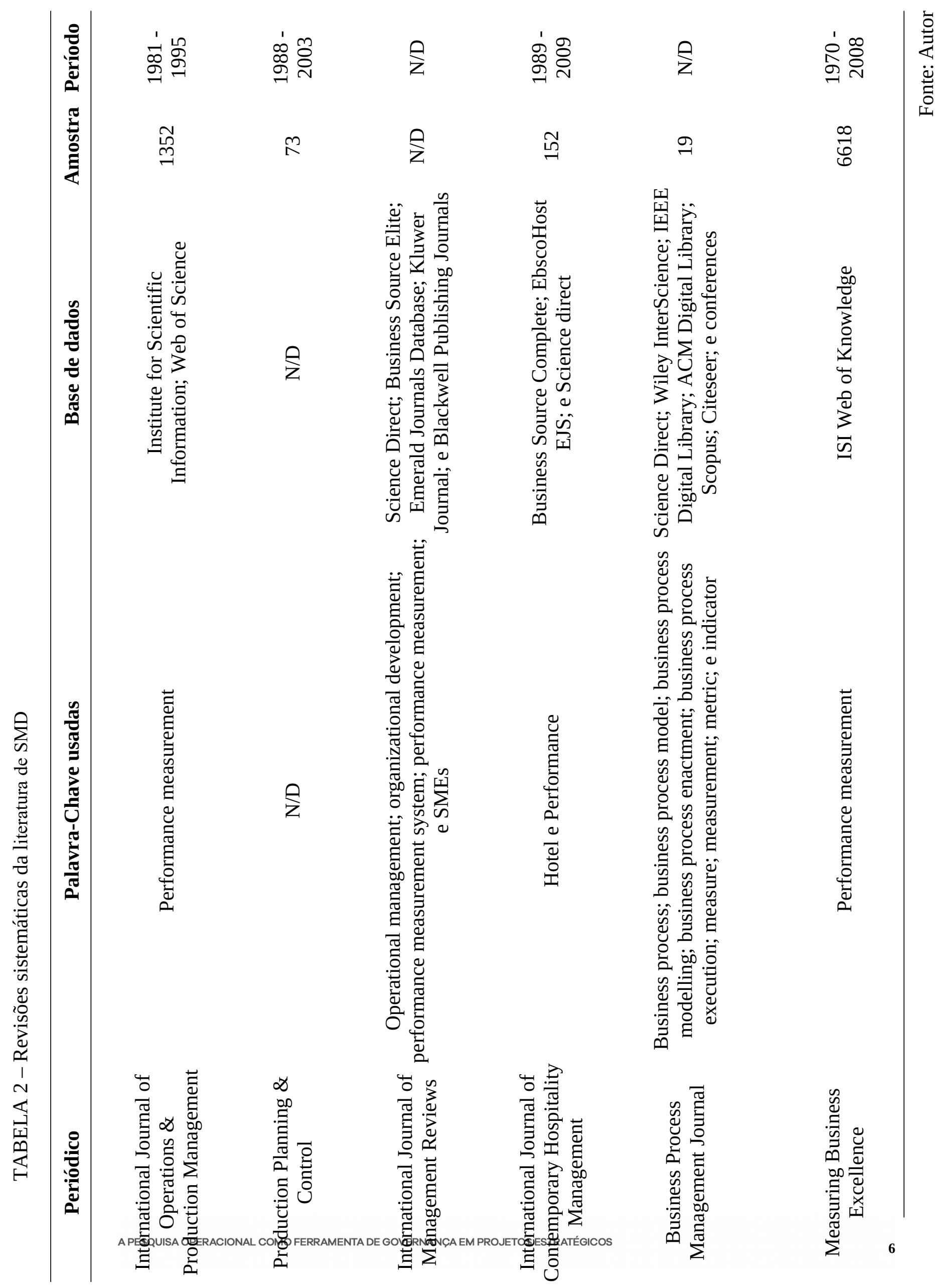




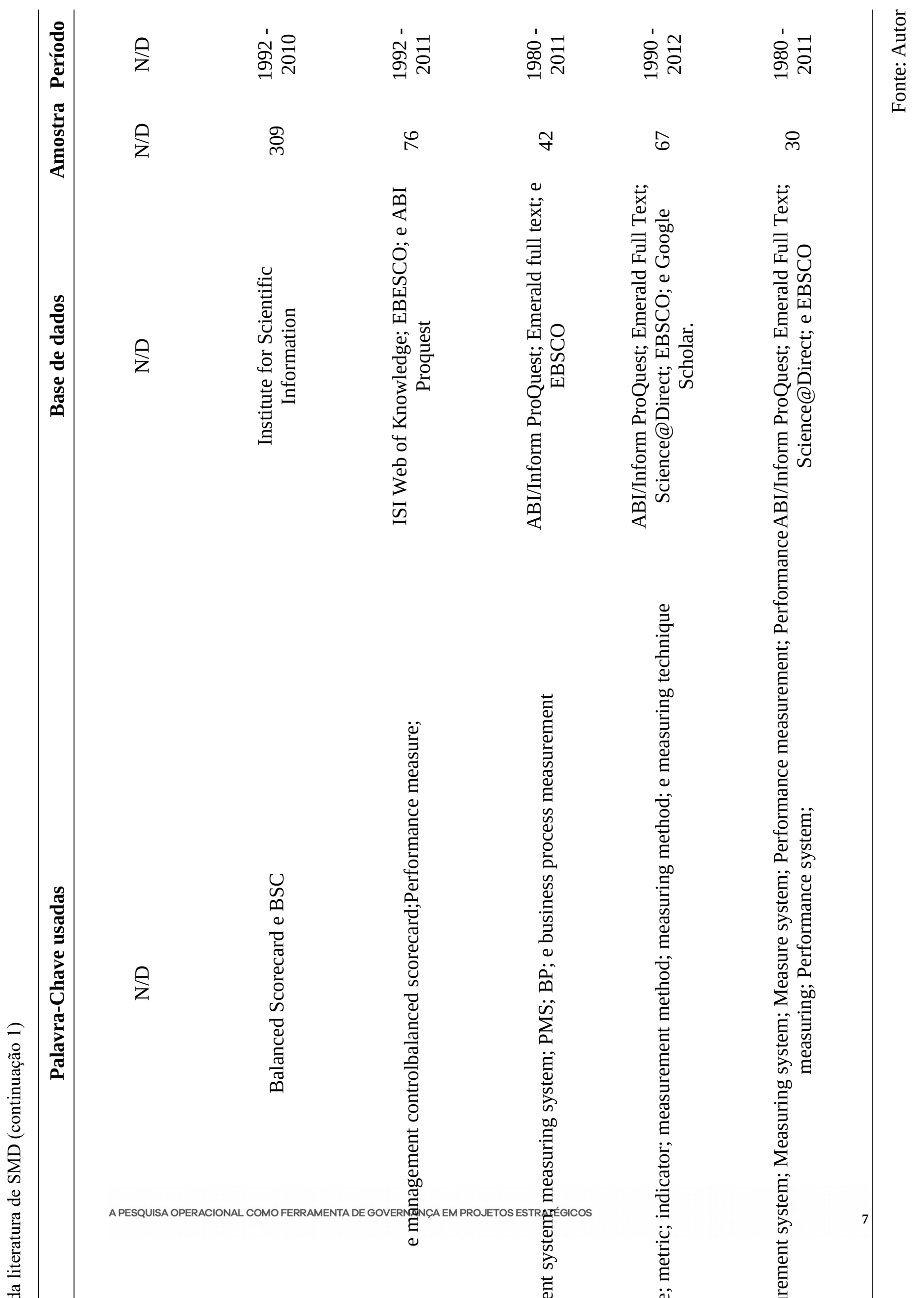


ํㅝำ

$\stackrel{\ominus}{\Sigma}$

$\stackrel{2}{Z}$

ํํ유ำ

극

$\stackrel{2}{\text { 乙 }}$

$\stackrel{\ominus}{\mathbf{Z}}$

の

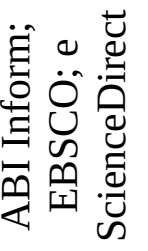

之

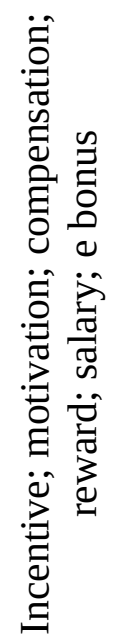

z

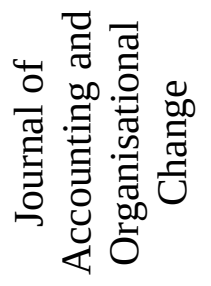

.0
0
0
0
0
0
అ

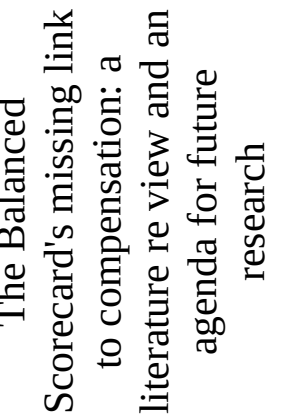

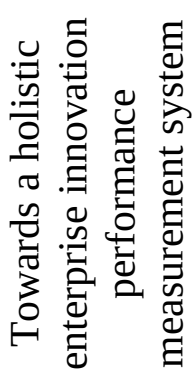

๑ $\overparen{\exists}$

ฮี 궁

艺

\#

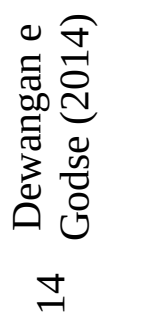

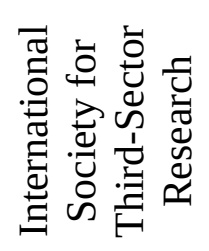

是
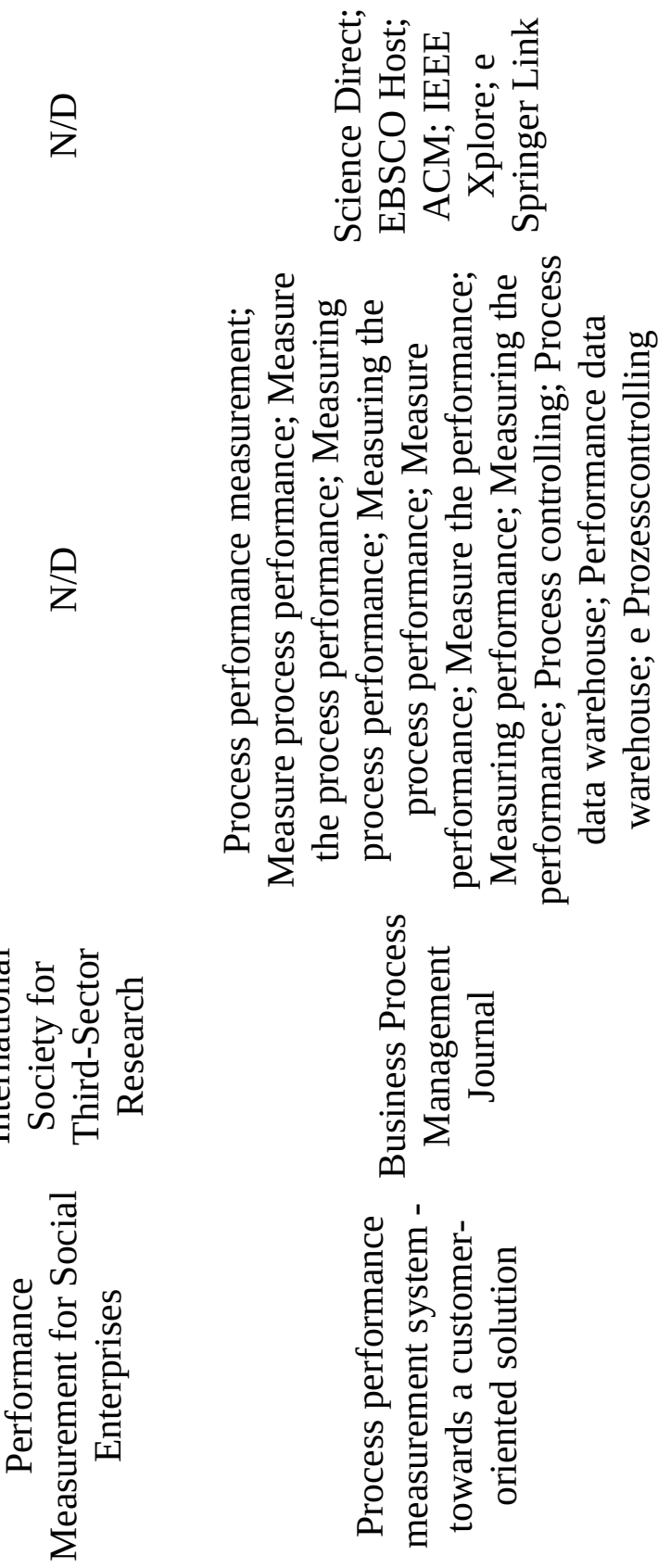

कं

तं

$\succsim \succcurlyeq$

苋

늠 


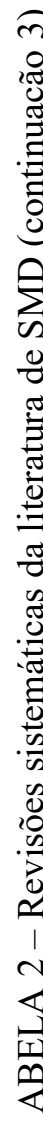

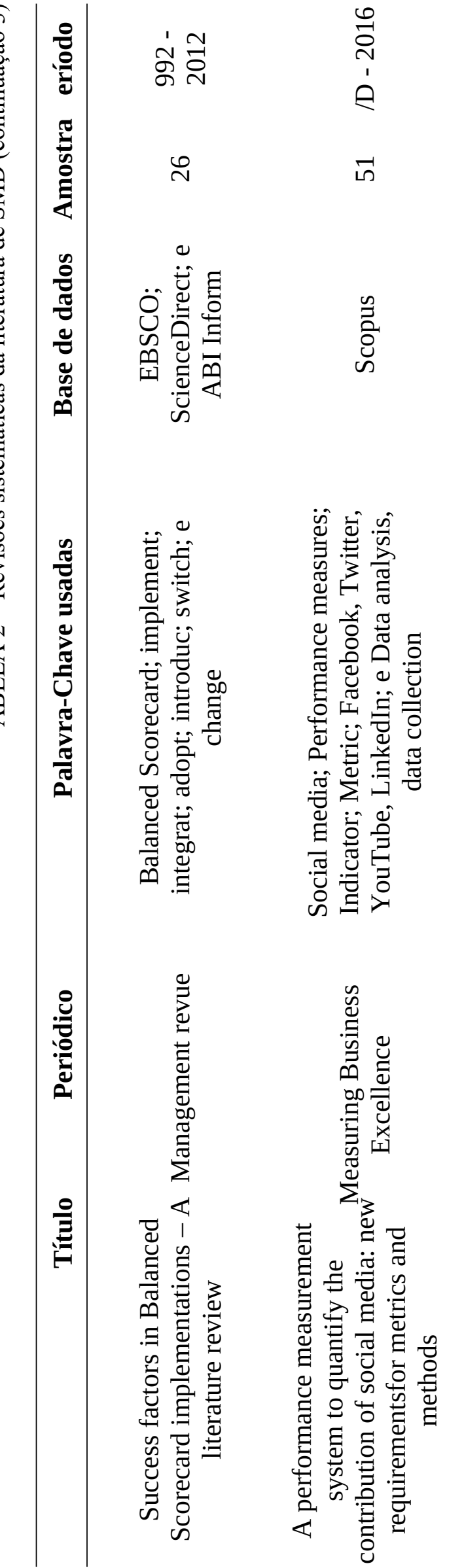

ঠ용 웅

$\stackrel{1}{\stackrel{0}{8}} \stackrel{0}{\circ}$

$\stackrel{\ominus}{\mathbf{Z}}$

$m$

$\stackrel{尺}{\mathrm{Z}}$

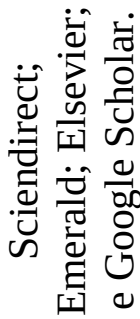

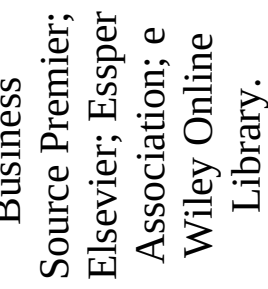

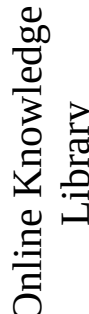

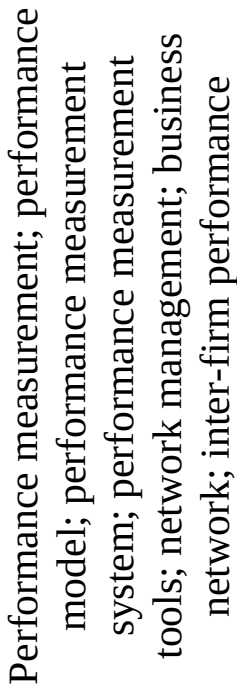

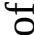

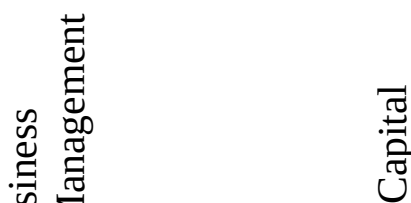

葛

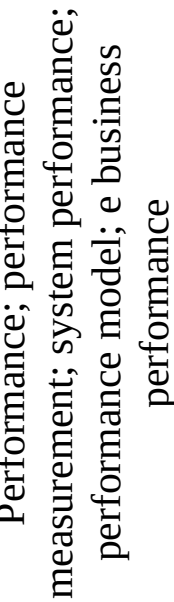

之

苍导

总: 䒿

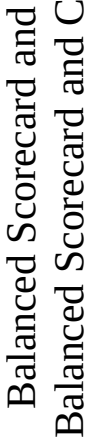

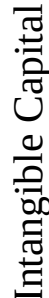

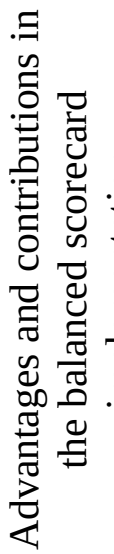




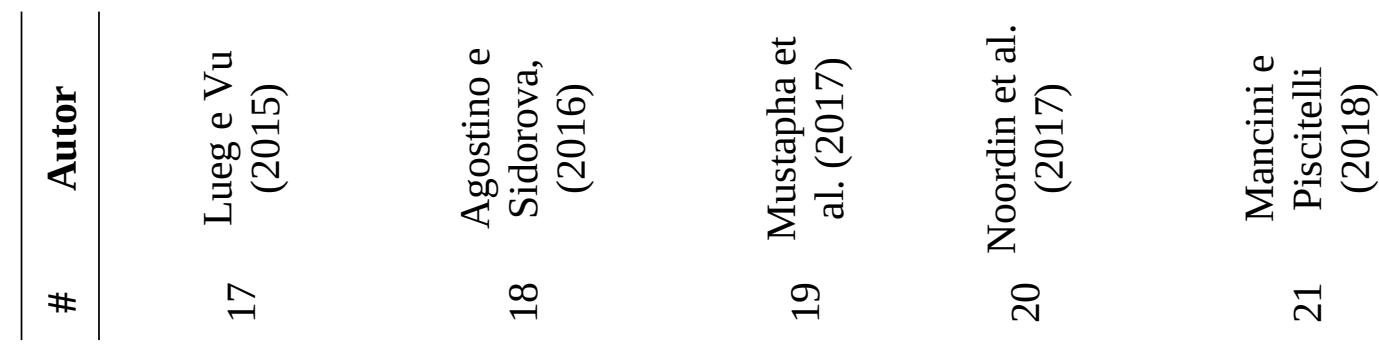

TABELA 3 - Objetivos das revisões sistemáticas da literatura de SMD.

\begin{tabular}{lll}
\hline$\#$ & Autor & Objetivo
\end{tabular}

1 Neely, (2005)

Realizar uma análise de citação/cocitação de publicações relacionadas à medição de desempenho com o intuito de atualizar o estado da pesquisa em medição de desempenho.

Franco-Santos e Examinar os fatores que afetam a habilidade das organizações realizarem o gerenciamenBourne (2005) to através das medições de desempenho.

3 Garengo et al. (2005)

Investigar a relação do SMD com pequenas e médias empresas.

Identificar o estado da arte, analisando os principais estudos nos últimos 20 anos em ges4 Sainaghi (2010) tão hoteleira. Além de usar o Balanced Scorecard para avaliar a coerência entre os resultados empíricos e os objetivos de pesquisa e sugerir uma agenda de pesquisa.

González et al. Atualizar o estado da arte em relação à mensuração do processo de negócio, a fim de pro(2010) por atividades de pesquisa inovadora. desenvolvidos para pequenas e médias empresas.

Nudurupati et al. (2011) SMD, a fim de evidenciar os gaps e propor uma agenda para pesquisas futuras. (2011) sim, corroborar a hipótese de que o BSC pode ser considerado um modelo teórico sólido que pode ser aplicável as empresas.

Franco-Santos et al. (2012)

Identificar quais são as consequências do sistema de medição de desempenho.

10 Choong (2013)

Identificar os fundamentos do SMD, a fim de verificar se são satisfeitos os requisitos do gerenciamento do processo de negócio. 
11 Choong (2013) Identificar os fatores necessários para conceituar os recursos de um sistema de medição de desempenho eficiente e eficaz.

12 Choong (2014) Verificar a contribuição das publicações sobre SMD para sua própria compreensão e se há necessidade de elaborar um modelo conceitual ou teórico para o SMD.

Fonte: Autor

TABELA 3 - Objetivos das revisões sistemáticas da literatura de SMD. (continuação)

\begin{tabular}{lll}
\hline$\#$ & Autor & Objetivo \\
\hline
\end{tabular}

13

Albertsen e Lueg

Buscar uma melhor compreensão entre o BSC e a compensação e como essa relação é (2014) apresentada em um BSC "completo" (conceito original).

Dewangan e God-

Enfatizar a distinção entre a medição de desempenho da invenção e da exploração, se (2014) além de usar uma taxonomia para os principais indicadores de desempenho da inovação.

Desenvolver uma abordagem para Social Enterprise que auxilie na medição de desem(2015) penho levando em consideração os aspectos sociais, ambientais e econômicos.

Formular uma proposta com recomendações para o design de um SMD holístico e ori(2015) entado ao cliente, utilizando quality function deployment (QFD).

Buscar um entendimento dos não-usuários do BSC que gostariam de implementá-lo, mas não o fazem por julgarem que não há uma experiência adequada ou não tem a capacidade de implementação adequada. rova (2016)

Fornecer um framework capaz de permitir uma compreensão sobre as métricas e métodos disponíveis que quantificam a contribuição das atividades de mídia social, considerando os impactos financeiros e não financeiros.

Propor um modelo de decisão para seleção de medidas de desempenho que devam ser incluídas no SMD.

Desenvolver um framework de contingência para delinear as abordagens que podem (2017) ser seguidas para instigar um sistema abrangente de medição de desempenho 
22 Quesado et al.

(2018)

Analisar as vantagens e contribuições da implementação do BSC.

Fonte: Autor

TABELA 4 - Gaps das revisões sistemáticas da literatura de SMD.

\begin{tabular}{ccl}
\hline$\#$ & Autor & Gap \\
\hline 1 & Neely (2005) & $\begin{array}{l}\text { Fases do ciclo evolutivo do SMD não estão bem delineadas; e carência de SMD dinâmico } \\
\text { que garante o foco no gerenciamento de desempenho. }\end{array}$
\end{tabular}

Franco-Santos e

Bourne (2005)

$3 \quad$ Garengo et al. (2005)

$4 \quad$ Sainaghi (2010)

González et al. (2010)

Taticchi et al. (2010)

Banchieri et al. (2011)

Nudurupati et al. (2011)

Franco-Santos et al. (2012)

10 Choong (2013)

11 Choong (2013)

12 Choong (2014)
Aprofundamento dos mapas estratégicos; confiabilidade e validação das medições de desempenho; definição de metas; relacionamento entre o SMD e outros sistemas; captação de informações não-financeiras; aplicação a novos contextos; e relacionamento com sistema de recompensas.

Poucos estudos teóricos e empíricos sobre a aplicação de SMD em pequenas e médias empresas.

Estudos no modelo Balanced Scorecard (BSC) sob a perspectiva financeira, sobretudo na utilização de variáveis financeiras independentes, desenvolvimento e inovação.

Poucos conceitos mensuráveis para as medidas dos processos de negócio e validação em um ambiente real.

Estabelecer condições necessárias para que as empresas extraiam valor das informações obtidas da medição de desempenho; e revisar os modelos, a fim de tratar com eficácia as questões culturais das pequenas e médias empresas.

Relacionamento entre os indicadores do BSC; considerar os aspectos de tempo e eficiência das medições na implementação do modelo; e refletir se o apoio da alta administração é necessário para sua implementação.

Justificativas de esforço investido ao longo do ciclo de vida do SMD frente aos benefícios da implementação do Sistema de Informação Gerencial; e explicar como o SMD evolui em resposta a mudanças no ambiente operacional interno e externo da organização.

Necessidade de aprofundar os estudos referentes ao impacto do sistema de medição de desempenho nos custos, cargas de trabalho, tensões e subjetividade.

Pouco foco nas medições em processos de negócio; objetivos não são claros; pouco foco no cliente; e carência de informações não-financeiras para o SMD

Características do SMD demasiadamente prescritiva, necessitando de um melhor esclarecimento, sobretudo pelas novas características baseadas em teoria normativa.

Pouco concordância na academia para descrição das atividades e características do SMD, 
além da escassez de consenso nas definições e termos sobre SMD.

Fonte: Autor

TABELA 4 - Gaps das revisões sistemáticas da literatura de SMD. (continuação)

\begin{tabular}{ccl}
\hline$\#$ & Autor & \multicolumn{1}{c}{ Gap } \\
\hline \multicolumn{4}{c}{ Albertsen e Lueg } & $\begin{array}{l}\text { Pouca evidência que indique quando um BSC tem sucesso ou não; não existe um cons- } \\
\text { truto para medir um BSC "completo"; carência de estudos no controle e avaliação do } \\
\text { (2014) }\end{array}$ & BSC; metodologia muito focada em estudos de caso.
\end{tabular}

Dewangan e Godse (2014)

Mais estudos para medição de desempenho da inovação.

Arena et al. Poucos estudos que abordem a medição de desempenho (financeira ou não-financeira) (2015) no setor social.

Wieland et al. (2015)

Integração entre requisitos dos clientes com o design do SMD.

17 Lueg e Vu (2015)

Há uma carência de estudos sobre fatores críticos de sucesso para implementação do BSC pertinentes aos aspectos qualitativos e meta-comunicativos.

Agostino e Sido- Dificuldade de manter atualizado as métricas e métodos de medição do SMD para mídia rova (2016) social, devido ao acelerado ritmo de evolução das mídias sociais.

Não há um consenso entre os
das de desempenho do SMD.

Noordin et al. (2017)

Poucos esforços em comparar o desempenho social e os seus impactos.

Mancini e Piscitelli (2018)

Entendimento da configuração do SMD e sua projeção em redes de negócios aplicados a diferentes contextos. BSC podem ser obtidas em novos contextos. 


\section{SPOLM2O19}

XIX SIMPÓSIO DE PESQUISA OPERACIONAL E LOGISTICA DA MARINHA

\section{CONCLUSÃO}

Com o aumento do número de publicações sobre SMD e, consequentemente, aumento de trabalhos de revisão da literatura, uma revisão terciária da literatura foi aplicada, a fim de alcançar os principais objetivos desta pesquisa - fornecendo uma visão integrada sobre as perspectivas das revisões sistemáticas da literatura em SMD e respondendo as perguntas de pesquisa P1 (Quais são os objetivos das revisões da literatura de SMD?) e P2 (Quais são os gaps remanescentes das revisões de literatura em SMD?).

Para o alcance do objetivo deste artigo foram analisados 22 papers. A amostra estudada corresponde ao intervalo de 2005 a 2018 e, através das análises dos trabalhos de revisão da literatura em SMD, foi observado que a maior parte dos estudos têm como objetivo aplicar um modelo que auxilie na definição de indicadores e proporcione uma compreensão do SMD, provando a sua eficiência e eficácia no contexto aplicado. Os resultados também apontam para uma carência de estudos que abordem outras fases do ciclo de vida do SMD além do design, o que tem sido frequentemente apontado como gap pelos pesquisadores. Além disso, há uma concentração de estudos na fase de design e nos modelos do Balanced Scorecard (BSC), dificultando o relacionamento do sistema de medição de desempenho com outros modelos.

\section{REFERÊNCIAS BIBLIOGRÁFICAS}

AGOSTINO, D.; SIDOROVA, Y. A performance measurement system to quantify the contribution of social media: new requirements for metrics and methods. Measuring Business Excellence, 20, n. 2, 2016. 38-51.

ALBERTSEN, O. A.; LUEG, R. The balanced scorecard's missing link to compensation: A literature review and an agenda for future research. Journal of Accounting \&

Organizational Change, 10, n. 4, 2014. 431-465.

ARENA, M.; AZZONE, G.; BENGO, I. Performance Measurement for Social Enterprises. International Journal of Voluntary and Nonprofit Organizations, 26, n. 2, 2015. 649672.

BANCHIERI, L. C.; PLANAS, F. C.; REBULL, M. V. S. What has been said, and what remains to be said, about the balanced scorecard? Zbornik Radova Ekonomskog Fakultet au Rijeci, 29, n. 1, 2011. 155-192.

BITITCI, U. et al. Performance Measurement: Challenges for Tomorrow. International Journal of Management Reviews, 14, n. 3, 2011. 305-327.

BRAZ, R. G. F.; SCAVARDA, L. F.; MARTINS, R. A. Reviewing and Improving Performance Measurement Systems: An Action Research. International Journal of Production Economics, 133, n. 2, 2011. 751-760.

CHOONG, K. K. Are PMS meeting the measurement needs of BPM? A literature review. Business Process Management Journal, 19, n. 3, 2013. 535-574.

CHOONG, K. K. Understanding the features of performance measurement system: a literature review. Measuring Business Excellence, 17, n. 4, 2013. 102-121.

CHOONG, K. K. Has this large number of performance measurement publications contributed to its better understanding? A systematic review for research and applications.

International Journal of Production Research, 52, n. 14, 2014. 4174-4197.

DEWANGAN, V.; GODSE, M. Towards a holistic enterprise innovation performance measurement system. Technovation, 34, n. 9, 2014. 536-545. 
FRANCO-SANTOS, M.; BOURNE, M. An Examination of the Literature Relating to Issues Affecting How Companies Manage through Measures. Production Planning \& Contro, 16, n. 2, 2005. 114-124.

FRANCO-SANTOS, M.; LUCIANETTI, L.; BOURNE, M. Contemporary performance measurement systems: a review of their consequences and a framework for research. Management Accounting Research, 23, n. 2, 2012. 79-119.

GARENGO, P.; BIAZZO, S.; BITITCI, U. Performance Measurement Systems in SMEs: A review of a research agenda. International Journal of Management Reviews, 7, n. 1, 2005. 25-47.

GONZÁLEZ, L. S. et al. Measurement in Business Processes: A Systematic Review. Business Process Management Journal, 16, n. 1, 2010. 114-134.

GONZÁLEZ-ALBO, B.; BORDONS, M. Articles vs. proceedings papers: Do they differ in research relevance and impact? A case study in the Library and Information Science field.

Journal of Informetrics, 5, n. 3, 2011. 369-381.

GOSHU, Y. Y.; KITAW, D. Performance measurement and its recent challenge: A literature review. International Journal of Business Performance Management, 18, 2017. 381-402.

GUNASEKARAN, A.; PATEL, C.; MCGAUGHEY, R. E. A framework for supply chain performance measurement. Int. J. Production Economics, 87, n. 3, 2004. 333-347.

GUNASEKARAN, A.; PATEL, C.; TIRTIROGLU, E. Performance measures and metrics in a supply chain environment. International Journal of Operations \& Production Management, 21, n. 1/2, 2001. 71-87.

GUTIERREZ, D. M. et al. Evolution of the Performance Measurement System in the Logistics Department of a Broadcasting Company: An Action Research. International Journal Production Economics, 160, Agosto 2015. 1-12.

KAPLAN, R.; NORTON, D. The balanced scorecard - Measures that drive performance. Harvard Business Review, 70, n. 1, 1992. 71-79.

KEATHLEY-HERRING, H. An Approach to Quantify the Factors That Affect Performance Measurement System Implementation. Engineering Management Journal, 29, n. 2, 2017. 63-73.

KENNERLY, M.; NEELY, A. A framework of the factors affecting the evolution of performance measurement systems. International Journal of Operations \& Production Management, 22, n. 11, 2002. 1222-1245.

LOHMAN, C.; FORTUIN, L.; WOUTERS, M. Designing a performance measurement system: A case study. European Journal of Operational Research, 156, n. 2, 2004. 267286.

LUEG, R.; VU, L. Success factors in Balanced Scorecard implementations - A literature review. Management Revue, 26, n. 4, 2015. 306-327.

MAESTRINI, V. et al. Supply chain performance measurement systems: A systematic review and research agenda. International Journal Production Economics, 183, 2017. 299-315.

MANCINI, D.; PISCITELLI, G. Performance measurement systems in business networks: a literature review. Int. J. Business Performance Management, 19, n. 1, 2018. 87-104. 
MUSTAPHA, N. M.; SOROOSHIAN, S.; AZIZAN, N. A. Performance Measures for Developing the Performance Measurement System: Systematic Literature Review Approach. Quality Management, 18, n. 156, 2017. 57-64.

NEELY, A. The evolution of performance measurement research - Developments in the last decade and a research agenda for the next. International Journal of Operations \& Production Management, 25, n. 12, 2005. 1264-1277.

NEELY, A. D.; GREGORY, M.; PLATTS, K. Performance measurement system design: a literature review and research agenda. International Journal of Operations and Production Management, 15, n. 4, 1995. 80-116.

NEELY, A. et al. Performance measurement system design: Should process based approaches be adopted? Int. J. Production Economics, 46-47, 1996. 423-431.

NOORDIN, N. H.; HARON, S. N.; KASSIM, S. Developing a comprehensive performance measurement system for waqf institutions. International Journal of Social Economics, 44, n. 7, 2017. 921-936.

NUDURUPATI, S. S. et al. State of the art literature review on performance measurement. Computers \& Industrial Engineering, 60, n. 2, 2011. 279-290.

QUESADO, P.; GUZMÁN, B. A.; RODRIGUES, L. L. Advantages and contributions in the balanced scorecard implementation. Intangible Capital, 14, n. 1, 2018. 186-201.

SAINAGHI, R. Hotel performance: state of the art. International Journal of Contemporary Hospitality Management, 22, n. 7, 2010. 920-952.

SEURING, S.; GOLD, S. Conducting content-analysis based literature reviews in supply chain management. Supply Chain Management: An International Journal, 17, n. 5, 2012. 544-555.

TATICCHI, P.; TONELLI, F.; CAGNAZZO, L. Performance Measurement and Management: A Literature Review and a Research Agenda. Measuring Business Excellence, 14, n. 1, 2010. 4-18.

THOMÉ, A. M. T.; SCAVARDA, L. F.; SCAVARDA, A. J. Conducting systematic literature review in operations management. Production Planning \& Control, 27, n. 5, 2016. 408-420.

TORGERSON, C. et al. Phonics: reading policy and the evidence of effectiveness from a systematic 'tertiary' review. Research Papers in Education, 2018.

WIELAND, U. et al. Process performance measurement system - towards a customeroriented solution. Business Process Management Journal, 21, n. 2, 2015. 312-331.

WOHLIN, C. Guidelines for Snowballing in Systematic Literature Studies and a Replication in Software Engineering. EASE '14 Proceedings of the 18th International Conference on Evaluation and Assessment in Software Engineering, 2014. 